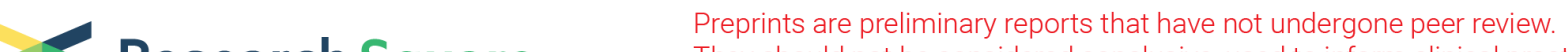 Research Square They should not be considered conclusive, used to inform clinical practice, or referenced by the media as validated information.
}

\section{Metformin Sensitizes Osteosarcoma Cells to Chemotherapy Through IGF-1R/miR-610/FEN1 Pathway}

\section{Suwei Dong}

The second affiliated hospital of Xuzhou medical university

\section{Yanbin Xiao}

The Third Affiliated Hospital of Kunming Medical University: Yunnan Cancer Hospital

\section{Ziqiang Zhu}

The Second Affiliated hospital of Xuzhou Medical University

\section{Xiang Ma}

The Third Affiliated Hospital of Kunming Medical University: Yunnan Cancer Hospital

\section{Zhuohui Peng}

The Third Affiliated Hospital of Kunming Medical University: Yunnan Cancer Hospital

\section{Jianping Kang}

The Third Affiliated Hospital of Kunming Medical University: Yunnan Cancer Hospital

\section{Biao Xie}

The Third Affiliated Hospital of Kunming Medical University: Yunnan Cancer Hospital

\section{Jianqiang Wang}

The Second Affiliated Hospital of Xuzhou Medical University

\section{Zhen Li}

The Second Affiliated Hospital of Xuzhou Medical University

\section{Yunqing Wang ( $\square$ kzwangyunqing@163.com )}

The Second Affiliated Hospital of Xuzhou Medical university https://orcid.org/0000-0002-7764-7481

\section{Research article}

Keywords: Osteosarcoma, Metformin, IGF-1R, FEN1, Chemosensitivity

Posted Date: January 18th, 2021

DOl: https://doi.org/10.21203/rs.3.rs-147084/v1

License: (c) (i) This work is licensed under a Creative Commons Attribution 4.0 International License. Read Full License 


\section{Abstract}

Background: Due to constitutive or acquired non-sensitive to cytotoxic agents, the prognosis of osteosarcoma remains unfavorable. It's has been proved that metformin could enhance the chemosensitivity of cancer cells to anticancer drugs. A novel finding states that IGF-1R involves in cancer chemoresistance, However, whether IGF-1R play a role in metformin-induced osteosarcoma chemosensitivity is incompletely understood. Hence, the current study aimed to elucidate the role of metformin in OS cell chemosensitivity modulation to identify the underlying mechanism of metformin regulating the IGF-1R/miR-610/FEN1 signaling.

Methods: Immunohistochemistry and qRT-PCR were used to evaluate the expression pattern of IGF-1R, miR-610 and FEN1 in osteosarcoma and paired normal tissues. Western blot and qRT-PCR were performed to determine changes in expression of key molecules in the IGF-1R/miR-610/FEN1 signaling pathway after various treatments. The direct modulation between miR-610 and FEN1 was monitored by luciferase reporter assay. Osteosarcoma cell sensitivity to chemotherapy was detected by MTS assay. In vivo experiments were conducted to further verify the role of the metformin in the chemosensitivity modulation of OS cells to ADM.

Results: We found that IGF-1R, miR-610 and FEN1 were abberently expressed in osteosarcoma, and participated in apoptosis modulation $(p<0.05)$. We found that this effect was abated by metformin treatment. Luciferase reporter assays confirmed that FEN1 is a direct target of miR-610. Moreover, we observed that metformin treatment decreased IGF-1R and FEN1, but elevated miR-610 expression. Metformin sensitized OS cells to cytotoxic agents, while overexpression of FEN1 compromised the sensitizing effects of metformin partly. Furthermore, metformin was observed to enforce the ADM treatment effect in nude mice xenograft models.

Conclusions: Overall, metformin enhanced the sensitivity of OS cells to cytotoxic agents via the IGF1R/miR-610/FEN1 signaling axis, highlighting the capacity of metformin as an adjunct to the chemotherapy of OS.

\section{Introduction}

Osteosarcoma (OS), the most frequent primary malignant bone tumor in children and the most common primary malignancy in adolescents apart from leukemia and lymphoma ${ }^{1}$. Currently, the combination of limb salvage and neoadjuvant chemotherapy are still major treatments for osteosarcoma patients, despite many efforts have contributed to treatment outcomes, its rate of death remains high by now ${ }^{2}$. Several high doses of anticancer drugs such as Adriamycin (ADM), cisplatin (DDP), and methotrexate (MTX) were most used to treat osteosarcoma ${ }^{3}$, but systemic toxicity and a risk of secondary cancer are concerns $^{4-6}$. Moreover, constitutive or acquired non-sensitive compromised the effective of the majority of chemotherapeutics ${ }^{7}$. 
Metformin (Met) is the most widely used drug in the treatment of type 2 diabetes with safe effects of insulin resistance reduction and blood glucose decrease ${ }^{8}$, has recently emerged as a potential of tumor prevention and treatment ${ }^{9-11}$. It has been reported that varied mechanisms underlying the antitumor effect of metformin, including inhibition of gluconeogenesis and oxidative phosphorylation, affecting cell growth, mobility, apoptosis, stemness and autophagy, etc ${ }^{9,12,13}$. More importantly, several studies have shown that metformin could enhance the cytotoxic effect in combine with various cytostatic drugs ${ }^{14,15}$. However, the mechanisms of these cooperative effects are still unclear.

Recent study showed that metformin could overcome primary resistance to EGFR-TKls with EGFR mutation via targeting Insulin-like growth factor 1 receptor (IGF-1R) signaling pathway ${ }^{16}$. IGF-1R is a transmembrane tyrosine kinase receptor in the insulin receptor family, which expression is upregulated in $\mathrm{OS}$, and the upregulation of IGF-1R is associated with surgical stage and metastasis. What's more, IGF-1R blockade increases chemo-sensitivity in multidrug-resistant osteosarcoma cell lines ${ }^{17}$.

The aim of the present study was to elucidate the potential role of metformin in OS cells chemosensitivity modulation and the underlaying mechanisms. We show that metformin enhances the anti-OS agents' cytotoxic effects by modulate IGF1R-miR610-FEN1 signaling pathway.

\section{Materials And Methods}

\section{Ethics statement}

The present study was approved by the Institutional Ethics Committee of The Third Affiliated Hospital of Kunming Medical University. Informed consent was obtained from each patient for the use of their tissues for research purposes, in accordance with the Declaration of Helsinki. Animal experiments were performed according to a strictly designed protocol, in accordance with the Guide for the Care and Use of Laboratory Animals published by the US National Institutes of Health. All efforts were made to ensure minimal suffering of the animal included in the study.

\section{Bioinformatics analysis}

Online websites GeneMANIA (http://genemania.org/) was employed to predict IGF-1R co-expression proteins, GEPIA (http://gepia.cancer-pku.cn/index.html) was utilized to analyze the expression correlation between IGF-1R and FEN1 in sarcoma tissues. Subsequently, miRTarBase (http://mirtarbase.mbc.nctu.edu.tw/php/index.php) and TargetScan (http://www.targetscan.org/) were employed to detect the miRNAs binding to FEN1. dbDEMC 2.0 data base (https://www.picb.ac.cn/dbDEMC/index.html) was performed to analysis the expression of miR-610 in tumor tissues (sarcoma, osteosarcoma subtype).

\section{Tissue samples}


Paired tumor and adjacent normal formalin-fixed paraffin-embedded (FFPE) samples were obtained from 66 patients, collected between 2015 and 2019. The miRNeasy FFPE Kit (Qiagen, Germany) was used to extract RNA from paraffin-embedded specimens. Patients with a diagnosis of relapse and who had received preoperative radiation, chemotherapy, or biotherapy were excluded from the study to avoid changes in tumor marker determination resulting from treatment.

\section{Cell culture, transfection, antibodies and reagents}

The human MG-63, U2OS and 143B cell lines were provided by American Type Culture Collection, ATCC. Cells were cultured in Dulbecco's modified Eagle's medium (DMEM) supplemented with $10 \%$ fetal bovine serum (Invitrogen; Thermo Fisher Scientific, USA.) and maintained at $37^{\circ} \mathrm{C}$ in a humidified incubator with $5 \% \mathrm{CO}_{2}$. The miR-610 mimic, miR-610 inhibitor, and negative control were designed and synthesized by Guangzhou RiboBio Co., Ltd (Guangzhou, China). The small interfering RNA (siRNA) targeting FEN1 and IGF-1R (si-FEN1, si-IGF1R) and negative control (NC) siRNA were also synthesized by Guangzhou RiboBio Co., Ltd. All plasmid constructs were verified by sequencing. Lipofectamine ${ }^{\circledR} 2000$ (Invitrogen, USA) was used for miRNA or siRNA transfection according to the manufacturer's protocols. Cells were harvested for subsequent experiments $48 \mathrm{~h}$ after transfection or anti-cancer drugs treatment. Metformin, ADM, DDP and MTX were purchased from Sigma, (St. Louis, MO, USA). Working dilutions of all drugs were prepared immediately before use. The antibodies to FEN1, IGF-1R, cleaved caspase-3 and GAPDH were obtained from Abcam.

\section{Luciferase reporter assay}

FEN1 3'-untranslated region (3'-UTR) containing the wild type or mutated miR-610 binding sequences were cloned into the pGL3-basic luciferase reporter vector (Promega, USA), named FEN1 WT and FEN1 MUT, respectively. Briefly, OS cells were seeded into a 24 -well plate at a density of $1 \times 10^{5}$ cells $/$ well. The cells were co-transfected with FEN1 WT or FEN1 MUT and the miR-610 mimic or NC miRNA by using Lipofectamine 2000 (Invitrogen, USA). The luciferase activity was measured using Dual-Luciferase Reporter Assay System (Promega, USA) and the activity of firefly luciferase was normalized to the corresponding Renilla luciferase activity.

\section{Reverse transcription-quantitative polymerase chain reaction (RT-qPCR)}

Total RNA was extracted using TRIzol Reagent (Invitrogen, USA) and purified with the RNeasy Maxi kit (Qiagen, Germany). The isolated total RNA was reverse transcribed using an miScript II RT Kit (Qiagen, Germany) to quantify both the mRNAs and miRNAs in the total RNA sample in a Roche Lightcycler 480 Real-Time PCR system (Roche Diagnostics, Switzerland). The relative miR-610 expression levels in tissue specimens and cells were calculated using the $2^{-\Delta \Delta C t}$ method $^{18}$, mRNA and miRNA expression were normalized to GAPDH and U6.. The primer used are depicted in Table 1. 
Table 1

Primer sequences for RT-qPCR.

\begin{tabular}{|lll|}
\hline Target & \multicolumn{2}{l|}{ Primer sequence (5'-3') } \\
\hline \multirow{2}{*}{ IGF-1R } & Forward: & 5'-TCATGCCTTGGTCTCCTTGT-3' \\
\cline { 2 - 3 } & Reverse: & 5'-TGCTTTGATGGTCAGGTTGC-3' \\
& Forward: & 5'-GTGAAGGCTGGCAAAGTCTA-3' \\
\cline { 2 - 3 } GAPDH & Reverse: & 5'-GCAGTCAGGTGTCGCATTA-3' \\
\cline { 2 - 3 } & Reverse: & 5'-TCAAAGGTGGAGGAGTGGGT-3' \\
\hline miR-610 & Forward: & 5'-TGCGCTGAGCTAAATGTGTCC-3' \\
\cline { 2 - 3 } & Reverse: & 5'-CAGTGCGTGTCGTGGAGT-3' \\
\hline U6 & Forward: & 5'-GCTTCGGCAGCACATATACTAAAAT-3' \\
\cline { 2 - 3 } & Reverse: & 5'-CGCTTCACGAATTTGCGTGTCAT-3' \\
\cline { 2 - 3 } \\
\end{tabular}

Table 2

Expression of IGF-1R and FEN1 in human OS and paired adjacent normal specimens.

\begin{tabular}{|lllll|}
\hline & Expression & Osterosarcoma & Normal & p value \\
\hline \multirow{2}{*}{ IGF-1R } & Positive & 46 & 5 & 0.000 \\
\cline { 2 - 4 } & Negative & 20 & 61 & \\
\multirow{2}{*}{ FEN1 } & Positive & 50 & 12 & 0.000 \\
\cline { 2 - 4 } & Negative & 16 & 54 & \\
\hline \multicolumn{2}{|l}{$p<0.05$} & Statistically significant difference. & \\
\hline
\end{tabular}

Table 3

Correlation analysis of IGF-1R and FEN1 in human OS specimens.

\begin{tabular}{|lccc|}
\hline & IGF-1R & & $p$ value \\
\hline & Positive & Negative & \\
\hline FEN1 & & & $p=0.009$ \\
\hline Positive & 39 & 11 & $r=0.319$ \\
\hline Negative & 7 & 9 & \\
\hline$p<0.05$ Statistically significant difference. \\
\hline
\end{tabular}




\section{Western blotting}

Protein was extracted with RIPA lysis buffer (89900, Pierce, USA) following the protocol. Protein concentrations were measured using the BCA assay (23227, Thermo, USA). For each sample, $50 \mu \mathrm{g}$ of protein lysate was loaded per well. Samples were electrophoresed on SDS-PAGE gels and transferred onto polyvinylidene fluoride (PVDF) membranes by electroblotting. The membranes were pretreated with $5 \%$ nonfat milk in TBS-T for $2 \mathrm{~h}$, followed by incubation with primary antibodies at $4{ }^{\circ} \mathrm{C}$ overnight. The following primary antibodies were used: anti-FEN1, ab17994; anti-IGF-1R, ab263903; and anti-Cleaved Caspase-3, ab49822, all from Abcam, USA. The membranes were then incubated with a horseradish peroxidase (HRP)-labeled secondary antibody (1:10,000, \#7076, Cell Signaling Technology, USA) for $1 \mathrm{~h}$. GAPDH was used as the internal loading control (1:1000, ab181602; Abcam). All the samples were assayed three times.

\section{Apoptosis analysis}

Cell apoptosis was analyzed using an Annexin V-fluorescein isothiocyanate (FITC) Apoptosis kit (BD Biosciences, USA) following the manufacturer's protocol. Cells were seeded into 24 -well plates $\left(1 \times 10^{5}\right.$ cells/well) and cultured for $24 \mathrm{~h}$. Cells were collected and washed twice with cold PBS, and stained with $1 \%$ FITC-labeled Annexin V and propidium iodide. After incubation apoptosis levels were evaluated using the FACS Aria system (BD Immunocytometry Systems, USA) and analyzed by Cell Quest software (Becton Dickinson Ltd). All experiments were performed three times independently.

\section{MTS assay}

To determine the cytotoxicity of metformin and the combined effect of metformin and chemotherapeutic agents. $5 \times 10^{3}$ cells were plated per well in 96 -well culture plates in $150 \mu$ of medium with serial doses of ADM, DDP or MTX, and 6 parallel wells were assigned to each group, as well as a negative control (without cells). $30 \mu \mathrm{l}$ of MTS substrate was added to each well, and then incubated for $2 \mathrm{hrs}$ in the dark, followed by ultraviolet irradiation for $15 \mathrm{~min}$. The absorbance at $490 \mathrm{~nm}$ was measured using plate reader (BMG Labtech, Germany). The concentrations required to inhibit growth by $50 \%$ (IC50) were calculated using the Bliss method ${ }^{19}$. All experiments were performed three times independently.

\section{Immunohistochemical (IHC) analysis}

Samples were processed for IHC analysis to determine FEN1 and IGF-1R expression levels and distribution patterns. $4 \mu \mathrm{m}$ paraffin-embedded tissue sections were mounted on charged glass slides and baked at $60^{\circ} \mathrm{C}$ for $2 \mathrm{~h}$. After deparaffinize in xylene, sections were microwave-treated for $10 \mathrm{~min}$ in citrate buffer ( $\mathrm{pH}$ 6.0) for antigen retrieval, and endogenous peroxidase activity was blocked by incubation in $0.3 \% \mathrm{H}_{2} \mathrm{O}_{2}$ solution. Rabbit polyclonal antibodies (FEN1, ab17994; IGF-1R ab263903, Abcam, USA) diluted 1:250 in phosphate-buffered saline (PBS) were used to detect the FEN1 or IGF-1R protein. After two washes in PBS, the slides were incubated with $A B C$ (Vector Laboratories, Burlingame, CA, USA), washed, overlaid with 3-30-diaminobenzidine (DAB; Dako Corporation, USA), and counterstained with hematoxylin. Human lung squamous carcinoma tissue was used as a positive control, while negative 
controls were obtained by replacing the primary antibody with non-immunized serum. Tissue was considered positive for FEN1 and IGF-1R protein expression if more than $10 \%$ of the tumor cells showed staining. All the slides were independently evaluated for protein expression by three different observers and slides with an incongruent grading were re-evaluated and a consensus was reached.

\section{Mice xenograft models}

4-5 weeks old athymic female BALB/c nude mice, were purchased from Beijing Vital River Laboratory Animal, Inc. (Beijing, China). Mice were randomly divided into 4 groups $\left(n=5 /\right.$ group). $5 \times 10^{7} 143 B$ cells were suspended in PBS were injected into the right flank of the nude mice. The length (a) and width (b) of the xenograft tumors were measured every two days with a Vernier caliper. Tumor volumes $\left(\mathrm{cm}^{3}\right)$ were calculated by the following formula: volume $=a b^{2} / 2$. When tumor volumes reach $0.1 \mathrm{~cm}^{3}$, mice were injected intraperitoneally with ADM (5 mg/kg, twice a week), metformin (250 mg/kg, once per day), or ADM (5 mg/kg, twice a week) plus metformin $\left(250 \mathrm{mg} / \mathrm{kg}\right.$, once per day) as stated in previous ${ }^{20,21}$. Drugs for injection were suspended in normal saline. Mice were sacrificed 21 days after inoculation, and tumors were excised and weighed.

\section{Statistics}

The correlation between immunocytochemical labeling of FEN1, IGF-1R and other clinical pathology parameters was analyzed by the $\chi^{2}$ test. Quantitative data are expressed as means \pm SD relative to the control value unless indicated otherwise. A $p$-value $<0.05$ was considered significant. All statistical analyses were performed in SPSS for Windows version 18.0 (SPSS Inc., Chicago, IL, USA). All assays were performed in triplicate.

\section{Results}

\section{IGF-1R/miR-610/FEN1 expression in OS tissues}

Firstly, we employed GeneMANIA to predict IGF-1R co-expression protein networks, our result showed an indirect interaction between Flap structure-specific endonuclease 1 (FEN1) and IGF-1R (Fig. 1A). FEN1 participates in numerous DNA processing pathways ${ }^{22}$, recent study uncovered that FEN1 inhibition sensitized cancer cells to drugs ${ }^{23}$. Furthermore, GEPIA analyze showed that IGF-1R expression was positively correlated with FEN1 ( $p<0.001, r=0.45$, Fig. 1B). Thus, we suspect IGF-1R might modulate OS cells chemo-sensitivity through the regulation of FEN1.

Then we examined the expression levels of IGF-1R and FEN1 in 66 OS and paired normal tissues, IGF-1R positive staining was confined mainly to the membrane and cytoplasm in OS tissues compared to a negatively stained normal tissue, and FEN1 expression mainly located in nucleus (Fig. 1C). Statistic results also confirmed the positive correlation between IGF-1R and FEN1 $(p=0.009, r=0.319$, Table. 2,3$)$. 
In order to study the underlaying mechanisms between IGF-1R and FEN1 interaction, miRTarBase and TargetScan predict miR-610 might bind to FEN1 3'-untranslated regions (3'-UTR). Besides, dbDEMC 2.0 database analysis showed that miR-610 expression was decreased in sarcoma, osteosarcoma subtype. More importantly, we examined the expression level of miR-610 in 20 OS and paired normal tissues, confirmed a lower expression of miR-610 in OS tissues (Fig. 1D). Based on these results, we suspect IGF1R/miR-610/FEN1 axis might participates in OS cells apoptosis and chemo-sensitivity modulation.

\section{IGF-1R/miR-610/FEN1 axis modulates OS cells apoptosis}

To further study the function of IGF-1R in OS, MG-63 and U2OS cells were transfected with IGF-1R si-RNA, both cell lines showed increased miR-610 and decreased FEN1 (Fig. 2A-D). Then we investigated the impact of IGF-1R silencing on cell apoptosis, FACS results showed that knockdown of IGF-1R induced OS cells apoptosis significantly (Fig. 2E).

miRTarBase and TargetScan prediction indicated that FEN1 was a potential target for miR-610. OS cell lines were transfected either with an miR-610 mimic or with an inhibitor. miRNA RT-PCR was utilized to assess miR-610 expression following transfection (Fig. 3A). While western blotting and real-time PCR showed that the miR-610 inhibitor increased FEN1 expression. In contrast, OS cells treated with the miR610 mimic exhibited the opposite trend (Fig. 3B, C).

In the dual-luciferase reporter assays, both FEN1 WT and MUT 3'-UTR sequences were structured based on potential binding sites. miR-610 overexpression greatly decreased luciferase activity when FEN1 WT was co-transfected into 293T cells. Whereas no alterations were detected upon co-transfection with MUTt-FEN1-3'UTR in cells. These data suggest that FEN1 is a direct target of miR-610 in OS cells (Fig. 3D).

Next, FEN1 si-RNA were transfected into OS cells (Fig. 4A). Then FACS results showed that knockdown of FEN1 induced OS cells apoptosis and elevated the expression of cleaved caspas-3 (Fig. 4B, C). These results indicated that inhibition of IGF-1/miR-610/FEN1 axis could induce OS cells apoptosis.

\section{Metformin regulates IGF-1R/miR-610/FEN1 signal and chemosensitivity of OS cells}

The mechanisms of metformin in cancer therapy are still not fully elucidated. When the OS cell lines were subjected to metformin treatment, their apoptosis rates were elevated by metformin $(p<0.05$ for both MG63 and U2OS. (Fig. 5A). Moreover, we proved that metformin treatment decreased the expression of IGF$1 \mathrm{R}$ and FEN1, but increased miR-610 expression (Fig. 5B, C).

To explore the effects of FEN1 in the anti-cancer activities of metformin, we constructed plasmids expressing FEN1. Transient transfection of the plasmids led to ectopic expression of FEN1 in OS cells. Then, we evaluated the sensitivity of MG-63 and U2OS cells to ADM, DDP and MTX when in addition with metformin. We found that anti-cancer drugs decreased the cell viability in OS cells but much more so in 
cells co-treated with metformin. More importantly, FEN1 overexpression compromised the sensitizing effects of metformin partly (Fig. 6A-C, Table 4). Collectively, these data suggest that IGF-1R/miR610/FEN1 signal involved in metformin mediated chemosensitivity of OS cells.

Table 4

The IC50 values of MG-63 and U20S by MTT assay.

\begin{tabular}{|c|c|c|c|c|}
\hline & MG-63 IC50 ( $(\mu \mathrm{M})$ & $p$ value & U2OS IC50 ( $(\mu \mathrm{M})$ & $p$ value \\
\hline ADM & $0.707 \pm 0.018$ & \multirow[t]{2}{*}{$p=0.025$} & $1.01 \pm 0.113$ & \multirow[t]{2}{*}{$p=0.031$} \\
\hline$A D M+$ Met & $0.551 \pm 0.03$ & & $0.596 \pm 0.039$ & \\
\hline DDP & $0.989 \pm 0.02$ & \multirow[t]{2}{*}{$p=0.017$} & $1.014 \pm 0.029$ & \multirow[t]{2}{*}{$p=0.00$} \\
\hline $\mathrm{DDP}+$ Met & $0.896 \pm 0.005$ & & $0.9 \pm 0.039$ & \\
\hline MTX & $35.277 \pm 0.971$ & \multirow[t]{2}{*}{$p=0.000$} & $32.79 \pm 0.691$ & \multirow[t]{2}{*}{$p=0.001$} \\
\hline MTX + Met & $12.3 \pm 0.403$ & & $10.297 \pm 0.499$ & \\
\hline
\end{tabular}

Metformin elevates chemo-sensitivity of OS cells in vivo

In order to examine the effect of metformin on OS cell tumorigenicity in an in vivo model, we inoculated 143B cells subcutaneously into the right flank of female athymic nude mice. When tumor volumes reach $0.1 \mathrm{~cm}^{3}$, mice were injected intraperitoneally with $A D M$, metformin, or ADM in combined with metformin for 21 days. Metformin combined therapy resulted in a markedly reduced growth rate $(p<0.01)$ and tumor weight $(p<0.05)$ compared with chemotherapy alone. (Fig. 7A, B) These data provide in vivo evidence that metformin contributes significantly to the chemo-sensitivity of OS cells.

\section{Discussion}

Despite of the success of chemotherapy for osteosarcoma, a majority of patients with OS experience recurrence and poor prognosis ${ }^{24}$. In recent years, several targeted- or immune-therapies have been evaluated in clinical trials, but these efforts did not prolong patients' lives significantly ${ }^{25-27}$. Preclinical studies aimed to uncover the underlaying biological pathways implicated in chemo-sensitivity are need 28-30. It's considered that IGF binding proteins are important regulators of bone metabolism and homeostasis ${ }^{31}$. Recently, researchers have confirmed that IGF-1R is involved in chemo-sensitivity modulation in osteosarcoma cell lines ${ }^{17,32}$. However, the underlaying mechanisms are still not fully demonstrated.

In present study, we predicted that FEN1 is co-expressed with IGF-1R. Then we showed that IGF-1R expression was positively correlated with FEN1. Further study confirmed that IGF-1R modulates FEN1 expresison by downregulates miR-610, which bind to FEN1 5'-UTR directly. Functional experiments 
demonstrated that IGF-1R/miR-610/FEN1 axis is involved in OS cells apoptosis modulation. In our previous study, we have shown that FEN1 is a key regulator of OS cells chemosensitivity ${ }^{33}$. Moreover, it's has been confirmed that metformin could increase TKIs sensitivity by targeting IGF-1R ${ }^{16}$. Thus, we suspect whether metformin impacts OS cells chemosensitivity via IGF-1R pathway.

As expected, we proved that metformin decreased the expression of IGF-1R and FEN1, but increased miR610. What's more, metformin increased OS cells chemosensitivity, FEN1 overexpression compromised the sensitizing effects of metformin partly. Finally, in vivo study also confirmed the chemo-sensitization effects of metformin.

Cytotoxic chemotherapy causes severe DNA damage directly by induction of breaks or indirectly due to the formation of reactive oxygen species (ROS) and ultimately induce cell death ${ }^{34,35}$. In responses to DNA damage, cells recruit DNA repair factors to upregulate mutagenic repair pathways that to maintain survival ${ }^{36}$. Thus, decrease DSB repair proteins expression could hypersensitising cells to DNA damage agents $^{37}$. It's has been proved that metformin could reduce the expression of DNA repair proteins ${ }^{38}$. FEN1 participates in the repair of DNA damage agent induced Top2-SSB complexes then maintain DNA stability ${ }^{39}$, disruption of FEN1 function leads to the accumulation of DNA double-strand breaks ${ }^{40}$. Thus, we suspect that metformin sensitizes OS cells to chemotherapy agents through the disruption of DNA damage repair processes.

\section{Conclusions}

Consequently, we are convinced that metformin enhances chemotherapy agents induced apoptosis via regulation of the IGF-1R/miR-610/FEN1 signaling axis in OS. More importantly, combine metformin with anticancer compounds is a tempting therapeutic strategy to enhance the anticancer effects, hopefully with clinical benefits to patients.

\section{Declarations}

\section{Acknowledgments}

This study was supported in part by grants from the National Natural Science Foundation of China (\#81760495), Applied basic research of Yunnan Science \& Technology Agency (Joint Funds of Yunnan Science \& Technology Agency and Kunming Medical University) (2018FE001-057).

\section{Disclosure of Potential Conflicts of Interest}

The authors declare that they have no competing interests.

\section{Consent for publication}

Not applicable. 


\section{Ethical Statement}

The study was approved by the ethics committee of the Third Affiliated Hospital of Kunming Medical University, and all patients gave written informed consent and authorization for use of biological specimens, in accordance with the Declaration of Helsinki.

\section{Availability of data and materials}

We declare that the materials described in the manuscript, including all relevant raw data, will be freely available to any scientist wishing to use them for non-commercial purposes, without breaching participant confidentiality.

\section{Authors' contributions}

Wang Yunqing, Li Zhen and Wang Jianqiang designed the study. Dong Suwei, Xiao Yanbin and Zhu Ziqiang collated the data, carried out data analyses and produced the initial draft of the manuscript. Ma Xiang performed the tissue test and analyzed and interpreted tissue data. Peng Zhuohui cultured the cells, prepared the protein and analyzed and interpreted PCR data. Kang Jianping performed the cell apoptosis test and analyzed and interpreted apoptosis data. Xie Biao performed xenograft models and interpreted in vivo data. All authors have read and approved the final submitted manuscript.

\section{References}

1. Damron TA, Ward WG, Stewart A. Osteosarcoma, chondrosarcoma, and Ewing's sarcoma: National Cancer Data Base Report. Clin Orthop Relat Res. 2007;459:40-7.

2. Simpson S, Dunning MD, de Brot S, Grau-Roma L, Mongan NP, Rutland CS. Comparative review of human and canine osteosarcoma: morphology, epidemiology, prognosis, treatment and genetics. Acta veterinaria Scandinavica. 2017;59(1):71.

3. Bacci G, Briccoli A, Rocca M, et al. Neoadjuvant chemotherapy for osteosarcoma of the extremities with metastases at presentation: recent experience at the Rizzoli Institute in 57 patients treated with cisplatin, doxorubicin, and a high dose of methotrexate and ifosfamide. Annals of oncology: official journal of the European Society for Medical Oncology. 2003;14(7):1126-34.

4. Czader M, Orazi A. Therapy-related myeloid neoplasms. Am J Clin Pathol. 2009;132(3):410-25.

5. Travis LB, Demark Wahnefried W, Allan JM, Wood ME, Ng AK. Aetiology, genetics and prevention of secondary neoplasms in adult cancer survivors. Nature reviews Clinical oncology. 2013;10(5):289301.

6. Bramwell VH. Osteosarcomas and other cancers of bone. Current opinion in oncology. 2000;12(4):330-6.

7. Li S, Sun W, Wang H, Zuo D, Hua Y, Cai Z. Research progress on the multidrug resistance mechanisms of osteosarcoma chemotherapy and reversal. Tumour biology: the journal of the International Society for Oncodevelopmental Biology Medicine. 2015;36(3):1329-38. 
8. Morris A. Diabetes. Systemic effects of metformin revealed. Nature reviews Endocrinology. 2017;13(10):562.

9. Suwei D, Liang Z, Zhimin L, et al. NLK functions to maintain proliferation and stemness of NSCLC and is a target of metformin. J Hematol Oncol. 2015;8:120.

10. Sambi M, Samuel V, Qorri B, et al. A Triple Combination of Metformin, Acetylsalicylic Acid, and Oseltamivir Phosphate Impacts Tumour Spheroid Viability and Upends Chemoresistance in TripleNegative Breast Cancer. Drug Des Devel Ther. 2020;14:1995-2019.

11. Anisimov VN. Metformin for Prevention and Treatment of Colon Cancer: A Reappraisal of Experimental and Clinical Data. Curr Drug Targets. 2016;17(4):439-46.

12. Schulten HJ. Pleiotropic Effects of Metformin on Cancer. International journal of molecular sciences 2018; 19(10).

13. Kawakita E, Yang F, Kumagai A, et al. Metformin mitigates DPP-4 inhibitor-induced breast cancer metastasis via suppression of mTOR signaling. Molecular cancer research: MCR; 2020.

14. Tang Z, Tang N, Jiang S, et al. The Chemosensitizing Role of Metformin in Anticancer Therapy. Anticancer agents in medicinal chemistry 2020.

15. Samuel SM, Varghese E, Koklesová L, Líšková A, Kubatka P, Büsselberg D. Counteracting Chemoresistance with Metformin in Breast Cancers: Targeting Cancer Stem Cells. Cancers 2020; 12(9).

16. Pan YH, Jiao L, Lin CY, et al. Combined treatment with metformin and gefitinib overcomes primary resistance to EGFR-TKIs with EGFR mutation via targeting IGF-1R signaling pathway. Biologics: targets therapy. 2018;12:75-86.

17. Duan Z, Choy E, Harmon D, et al. Insulin-like growth factor-I receptor tyrosine kinase inhibitor cyclolignan picropodophyllin inhibits proliferation and induces apoptosis in multidrug resistant osteosarcoma cell lines. Mol Cancer Ther. 2009;8(8):2122-30.

18. Livak KJ, Schmittgen TD. Analysis of relative gene expression data using real-time quantitative PCR and the 2(-Delta Delta C(T)) Method. Methods (San Diego, Calif) 2001; 25(4): 402-8.

19. Shi Z, Liang YJ, Chen ZS, et al. Reversal of MDR1/P-glycoprotein-mediated multidrug resistance by vector-based RNA interference in vitro and in vivo. Cancer Biol Ther. 2006;5(1):39-47.

20. Huang J, Ni J, Liu K, et al. HMGB1 promotes drug resistance in osteosarcoma. Cancer research. 2012;72(1):230-8.

21. Bao X, Zhao L, Guan H, Li F. Inhibition of LCMR1 and ATG12 by demethylation-activated miR-570-3p is involved in the anti-metastasis effects of metformin on human osteosarcoma. Cell death disease. 2018;9(6):611.

22. Balakrishnan L, Bambara RA. Flap endonuclease 1. Annual review of biochemistry. 2013;82:119-38.

23. Guo E, Ishii Y, Mueller J, et al. FEN1 endonuclease as a therapeutic target for human cancers with defects in homologous recombination. Proc Natl Acad Sci USA. 2020;117(32):19415-24. 
24. Otoukesh B, Boddouhi B, Moghtadaei M, Kaghazian P, Kaghazian M. Novel molecular insights and new therapeutic strategies in osteosarcoma. Cancer cell international. 2018;18:158.

25. Hattinger CM, Patrizio MP, Magagnoli F, Luppi S, Serra M. An update on emerging drugs in osteosarcoma: towards tailored therapies? Expert opinion on emerging drugs 2019; 24(3): 153-71.

26. Wu CC, Livingston JA. Genomics and the Immune Landscape of Osteosarcoma. Adv Exp Med Biol. 2020;1258:21-36.

27. Hattinger $\mathrm{CM}$, Fanelli M, Tavanti $\mathrm{E}$, et al. Doxorubicin-resistant osteosarcoma: novel therapeutic approaches in sight? Future oncology. (London England). 2017;13(8):673-7.

28. Wang G, Sun $M$, Jiang $Y$, et al. Anlotinib, a novel small molecular tyrosine kinase inhibitor, suppresses growth and metastasis via dual blockade of VEGFR2 and MET in osteosarcoma. International journal of cancer. 2019;145(4):979-93.

29. Yu Z, Li N, Jiang K, Zhang N, Yao LL. MiR-100 up-regulation enhanced cell autophagy and apoptosis induced by cisplatin in osteosarcoma by targeting mTOR. Eur Rev Med Pharmacol Sci. 2018;22(18):5867-73.

30. Zhao C, Zhang Q, Yu T, Sun S, Wang W, Liu G. Hypoxia promotes drug resistance in osteosarcoma cells via activating AMP-activated protein kinase (AMPK) signaling. Journal of bone oncology. 2016;5(1):22-9.

31. Conover CA. Insulin-like growth factor-binding proteins and bone metabolism. American journal of physiology Endocrinology metabolism. 2008;294(1):E10-4.

32. Wang $\mathrm{YH}$, Xiong J, Wang SF, et al. Lentivirus-mediated shRNA targeting insulin-like growth factor-1 receptor (IGF-1R) enhances chemosensitivity of osteosarcoma cells in vitro and in vivo. Molecular and cellular biochemistry 2010; 341(1-2): $225-33$.

33. Dong S, Xiao Y, Ma X, et al. miR-193b Increases the Chemosensitivity of Osteosarcoma Cells by Promoting FEN1-Mediated Autophagy. OncoTargets therapy. 2019;12:10089-98.

34. Reuvers TGA, Kanaar R, Nonnekens J. DNA Damage-Inducing Anticancer Therapies: From Global to Precision Damage. Cancers 2020; 12(8).

35. Lozano R, Castro E, Aragón IM, et al. Genetic aberrations in DNA repair pathways: a cornerstone of precision oncology in prostate cancer. British journal of cancer 2020.

36. Jeggo PA, Löbrich M. How cancer cells hijack DNA double-strand break repair pathways to gain genomic instability. Biochem J. 2015;471(1):1-11.

37. Brown JS, O'Carrigan B, Jackson SP, Yap TA. Targeting DNA Repair in Cancer: Beyond PARP Inhibitors. Cancer discovery. 2017;7(1):20-37.

38. Jeong YK, Kim MS, Lee JY, Kim EH, Ha H. Metformin Radiosensitizes p53-Deficient Colorectal Cancer Cells through Induction of G2/M Arrest and Inhibition of DNA Repair Proteins. PloS one. 2015;10(11):e0143596.

39. Kametani Y, Takahata C, Narita T, Tanaka K, Iwai S, Kuraoka I. FEN1 participates in repair of the 5'phosphotyrosyl terminus of DNA single-strand breaks. Carcinogenesis. 2016;37(1):56-62. 
40. Ward TA, McHugh PJ, Durant ST. Small molecule inhibitors uncover synthetic genetic interactions of human flap endonuclease 1 (FEN1) with DNA damage response genes. 2017; 12(6): e0179278.

\section{Figures}

Figure 1

A

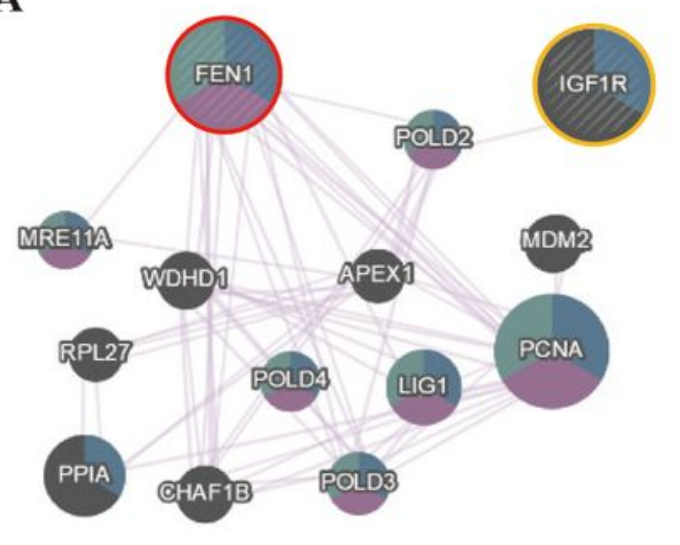

C

IGF-1R
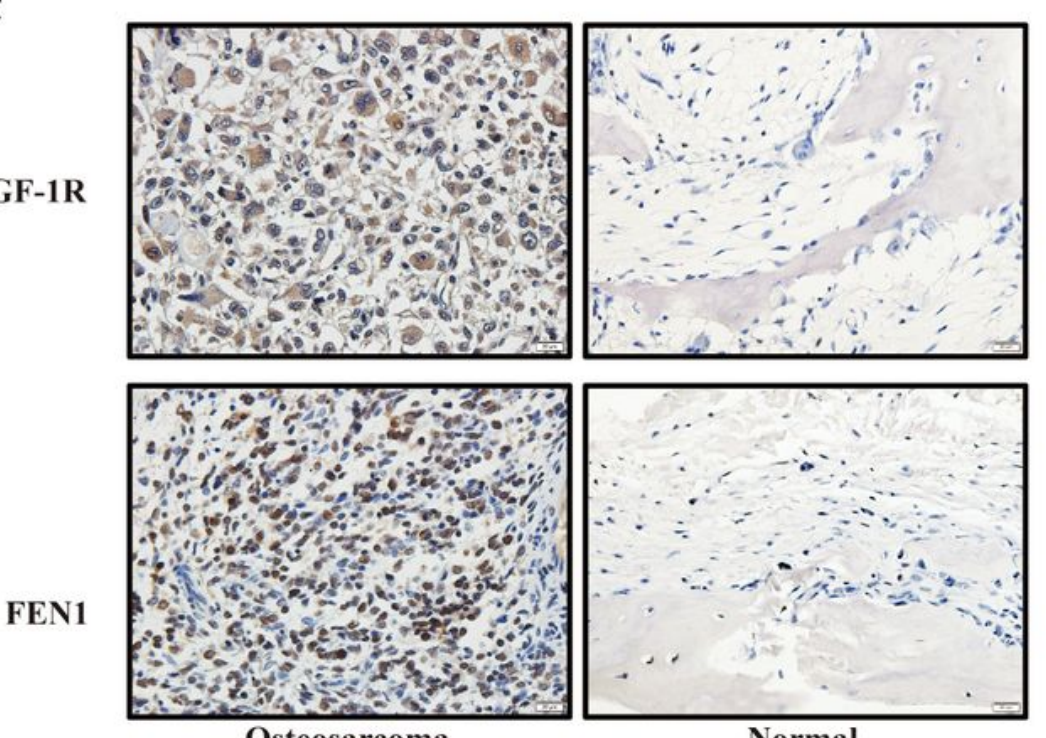

Osteosarcoma

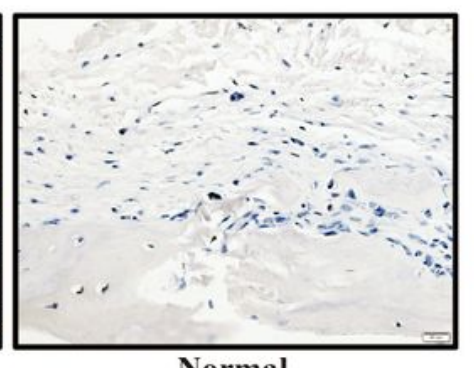

Normal

D

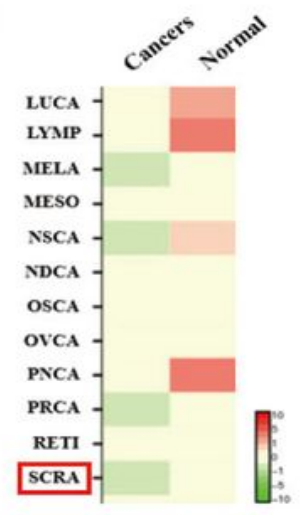

E

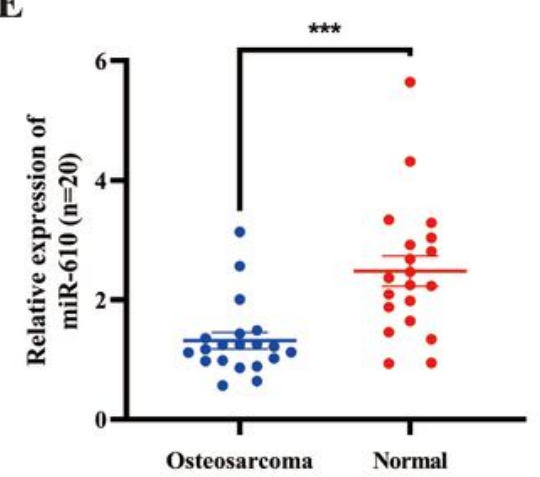

B

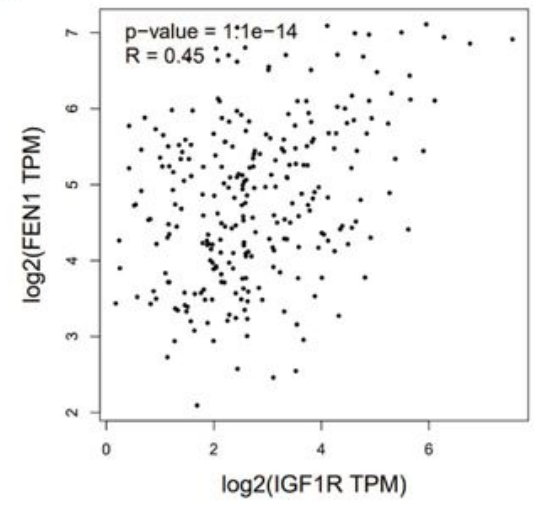

Figure 1 
Expression patterns of IGF-1R, miR-610 and FEN1 in OS. (A, B) Data base analysis showed the correlation between IGF-1R and FEN1. (C) Immunohistochemical staining of IGF-1R and FEN1 in OS and paired adjacent normal specimens. (D, E) Data base analysis and RT-qPCR measured miR-610 expression in OS tissues. ${ }^{*} p<0.05, * \star p<0.01, * \star * p<0.001$ vs. control.

\section{Figure 2}

A

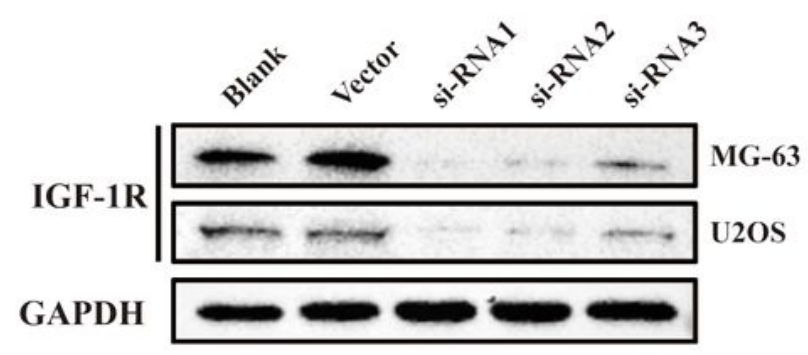

B

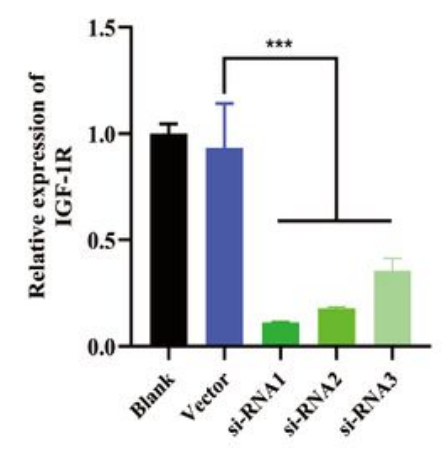

C

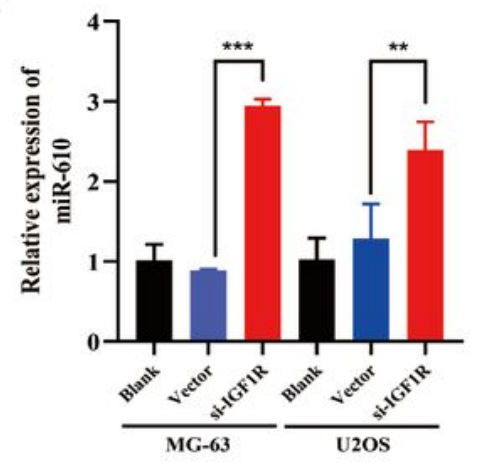

D

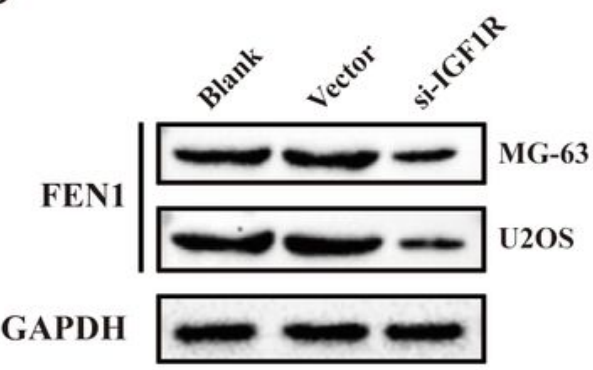

$\mathbf{E}$
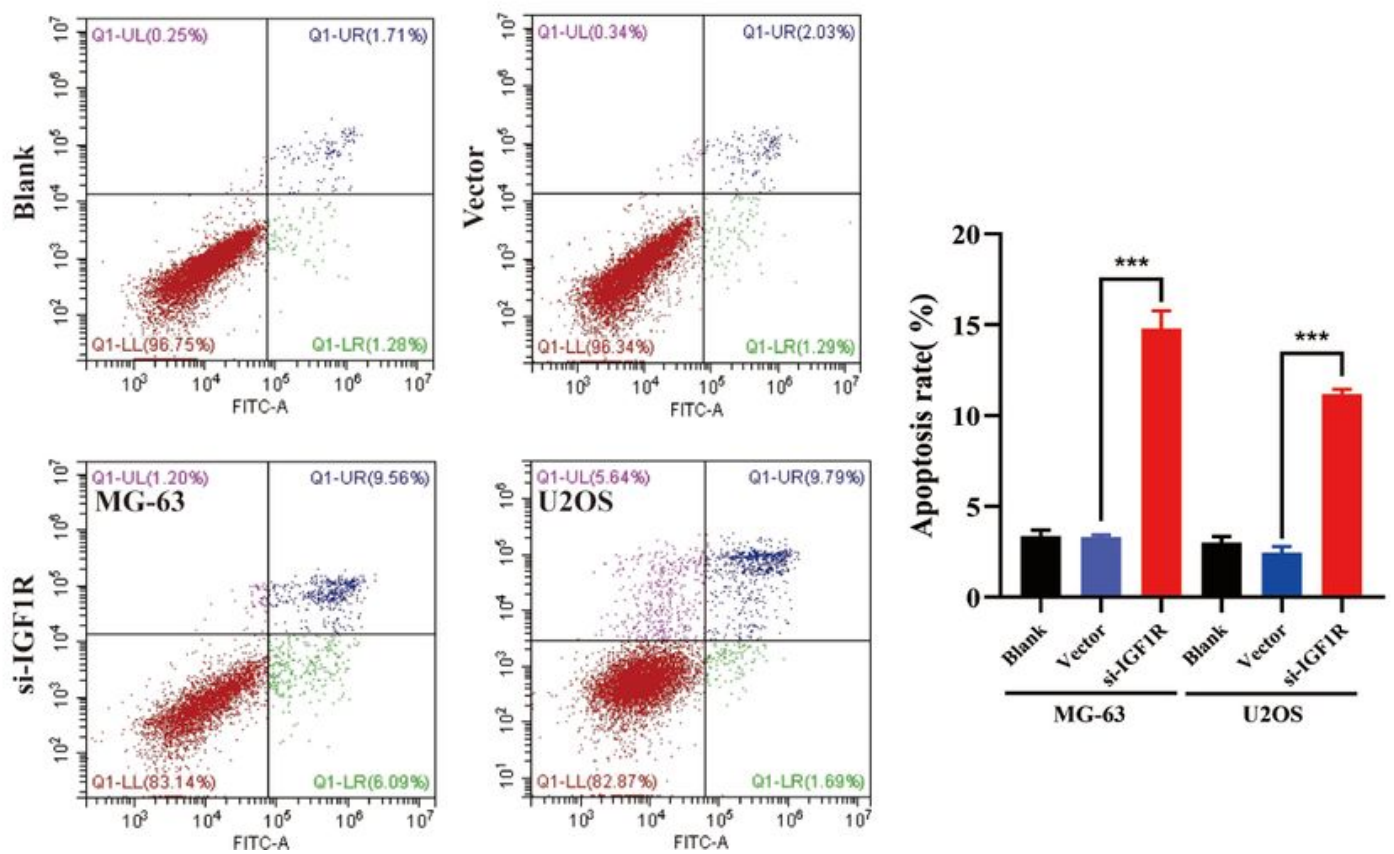

Figure 2 
IGF-1R modulates miR-610/FEN1 expression and apoptosis of OS cells. (A-D) The expression levels of miR-610 and FEN1 under altered IGF-1R expression. (E) Apoptosis of OS cells was determined by flow cytometry upon treatment with IGF-1R si-RNA. ${ }^{*} p<0.05$, ${ }^{* *} p<0.01$, ${ }^{* \star *} p<0.001$ vs. control.

Figure 3

A

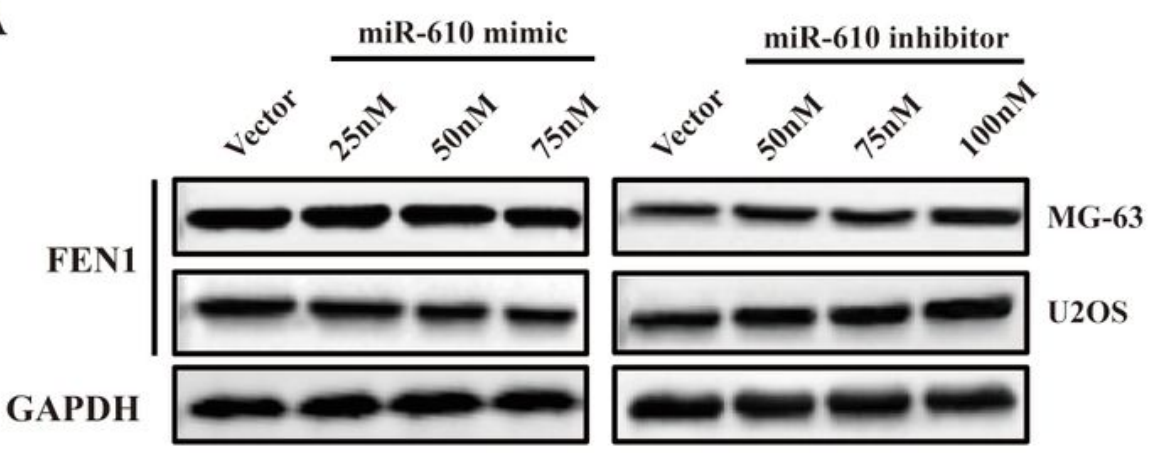

B

C
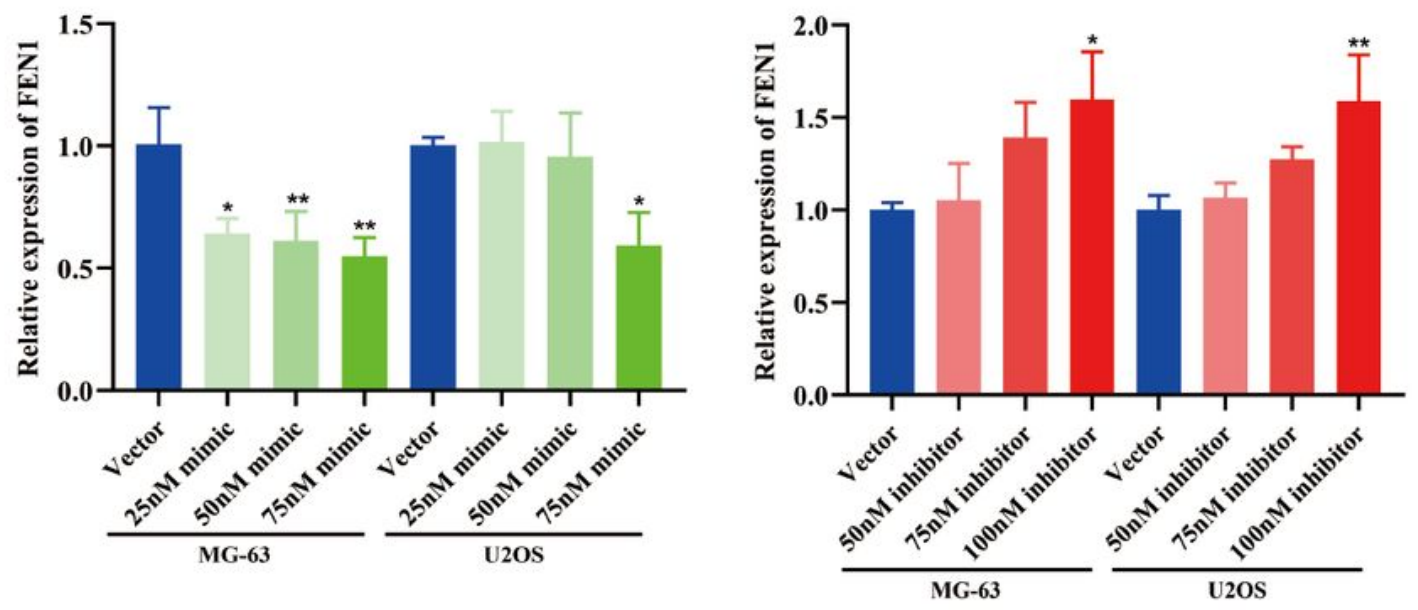

D
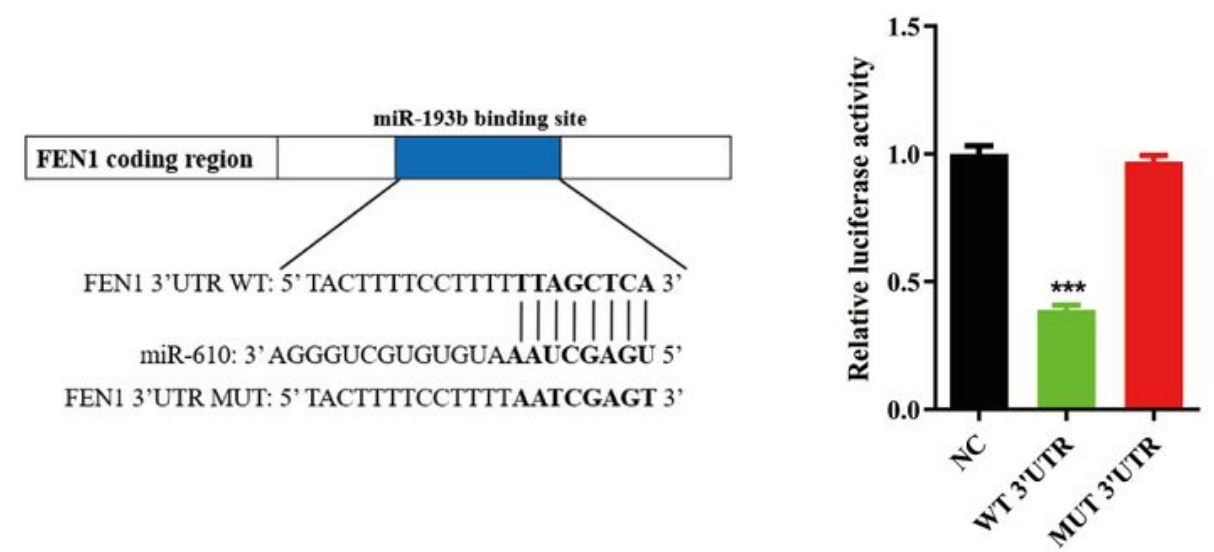

Figure 3

FEN1 is a direct target of miR-610. (A-C) miR-610 modulates FEN1 expression, the level of FEN1 protein and mRNA expression in OS cells transfected with either a mimic or an inhibitor of miR-610. (D) The miR- 
610 binding site in the 3'-UTR of FEN1 and matched mutations. A luciferase reporter assay was conducted in OS cells to verify the interaction between miR-610 and the FEN1 binding site. * $p<0.05$, ** $p$ $<0.01,{ }^{* *} \mathrm{p}<0.001$ vs. control.

\section{Figure 4}

A

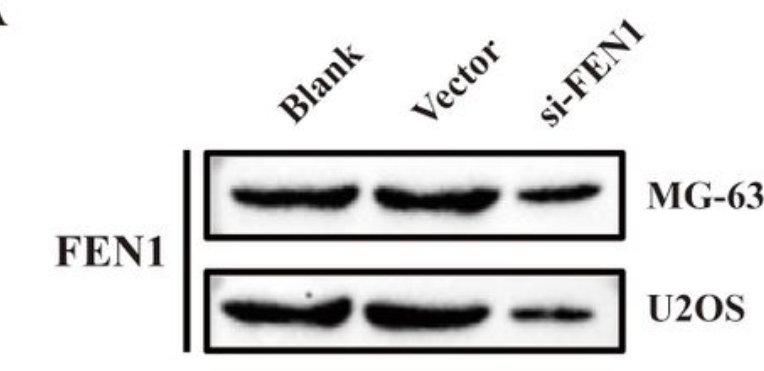

GAPDH

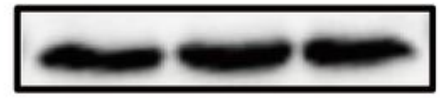

$\mathbf{C}$

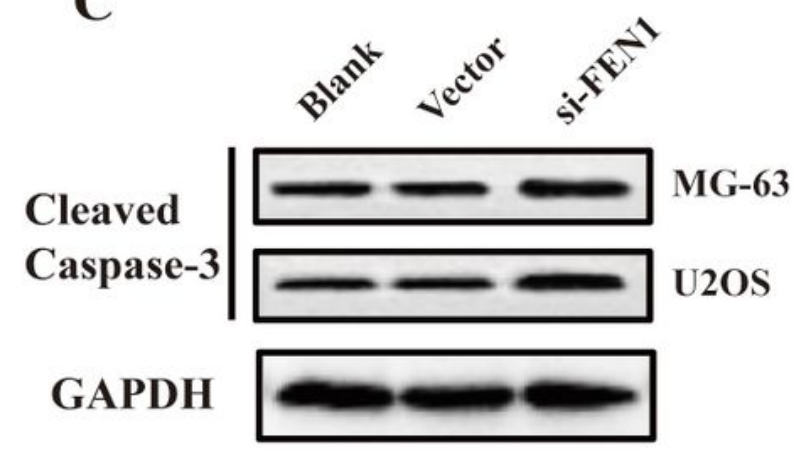

B
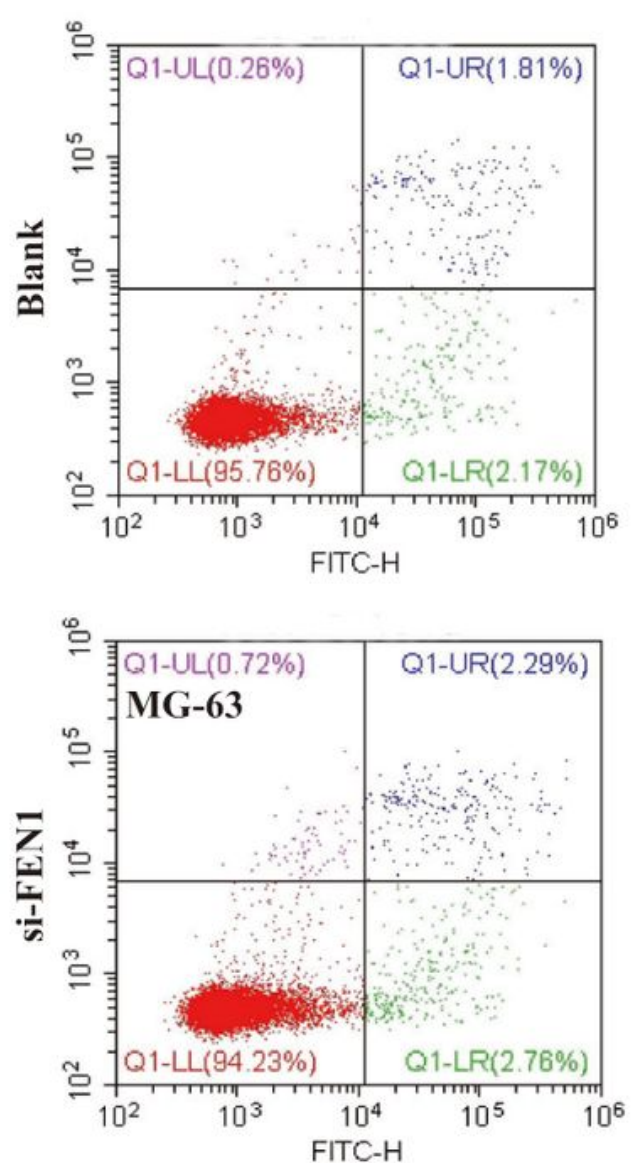
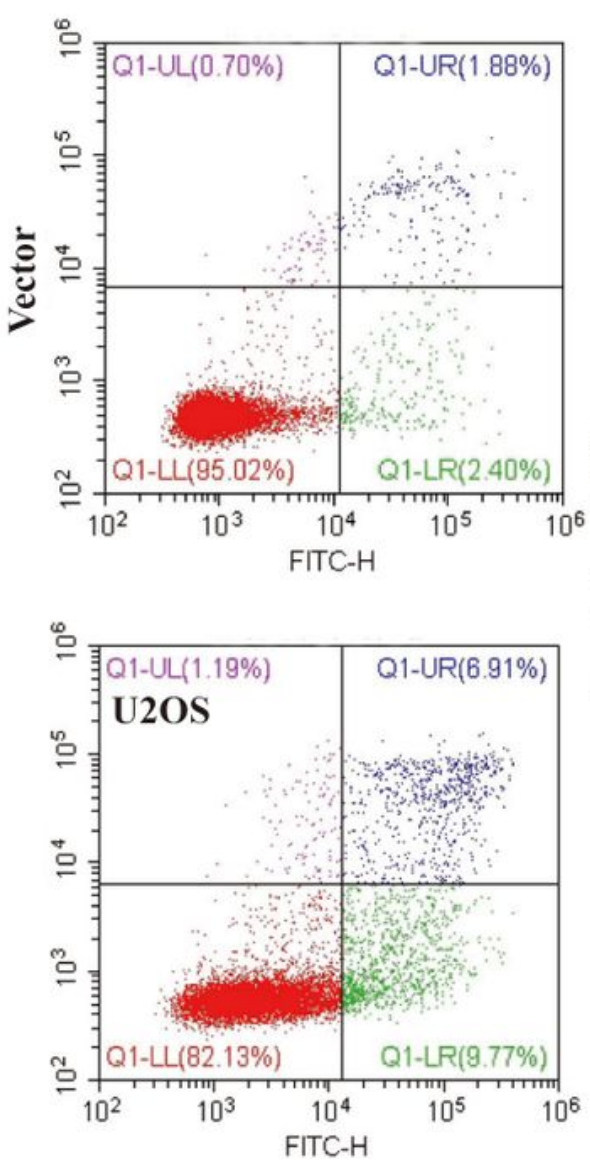

Figure 4

FEN1 modulates apoptosis of OS cells. (A-B) Apoptosis of OS cells was determined by flow cytometry upon treatment with FEN1 si-RNA. (C) The expression levels of Cleaved-Caspase3 under altered FEN1 
expression. * $p<0.05$, ** $p<0.01$, *** $p<0.001$ vs. control.

Figure 5

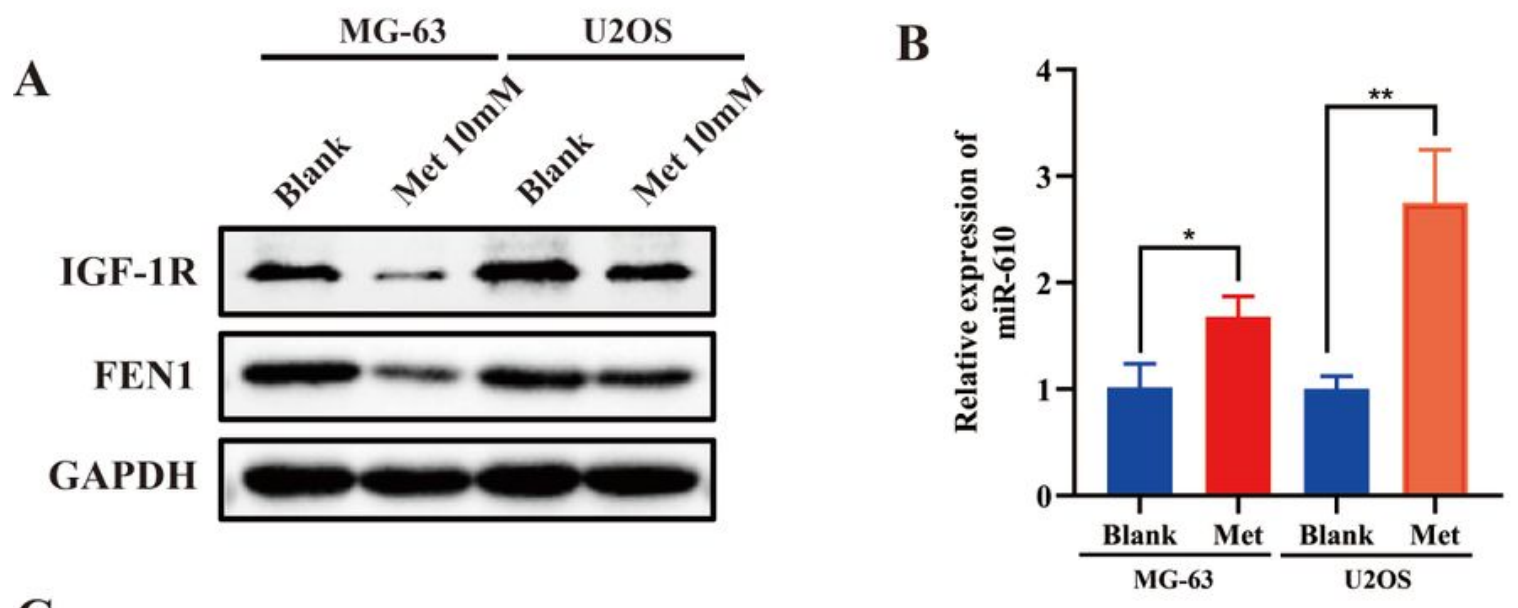

C

MG-63
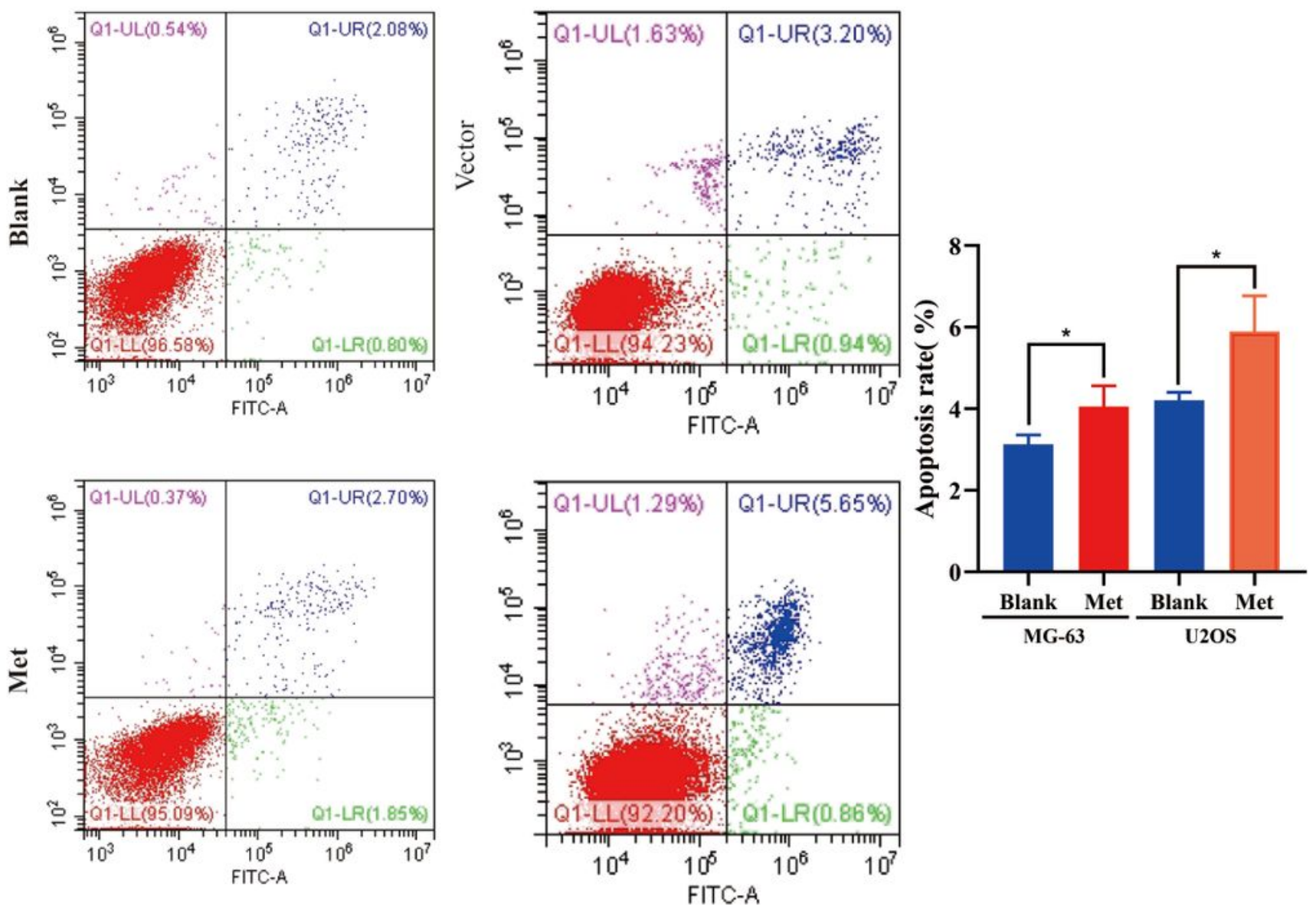

Figure 5

Metformin impacts IGF-1R/miR-610/FEN1 axis expression and OS cells apoptosis. (A, B) The expression levels of IGF-1R, miR-610 and FEN1 after metformin treatment. (C) Apoptosis of OS cells was determined by flow cytometry after metformin treatment. * $p<0.05$, ${ }^{* \star} p<0.01$, ${ }^{\star \star *} p<0.001$ vs. control. 
A

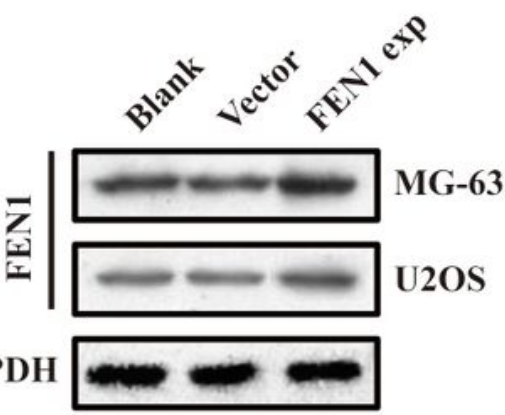

C

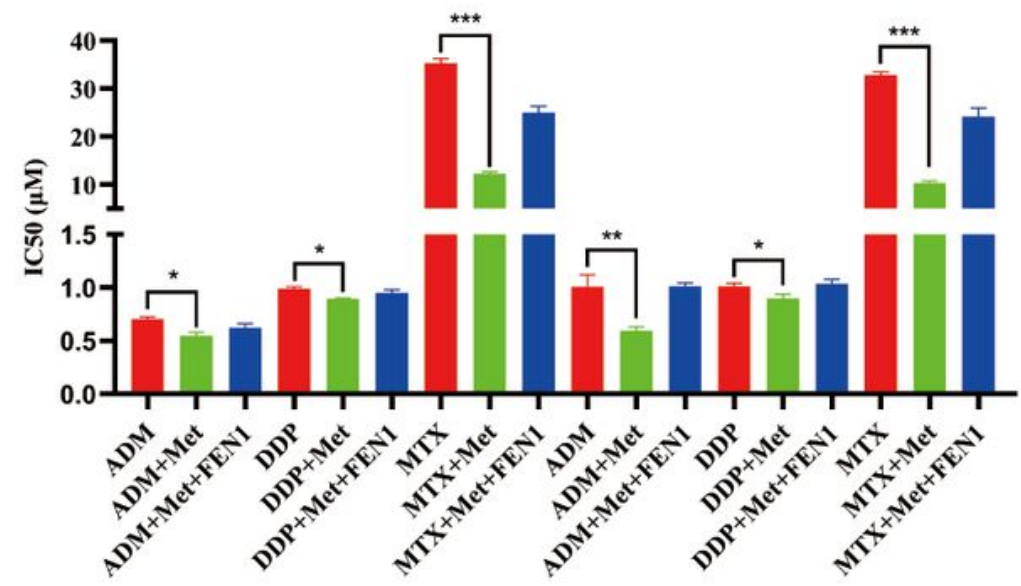

B

ADM
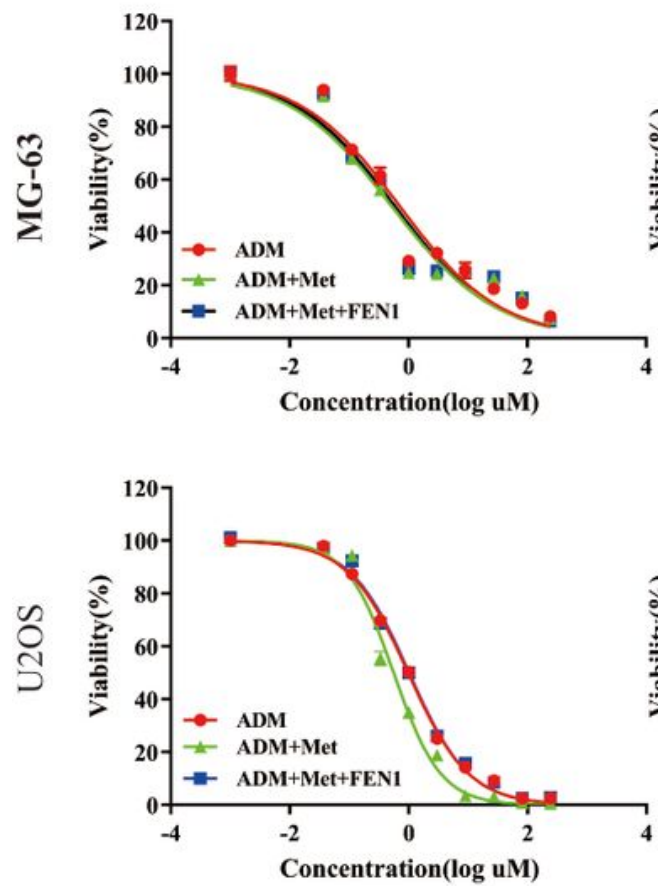

DDP
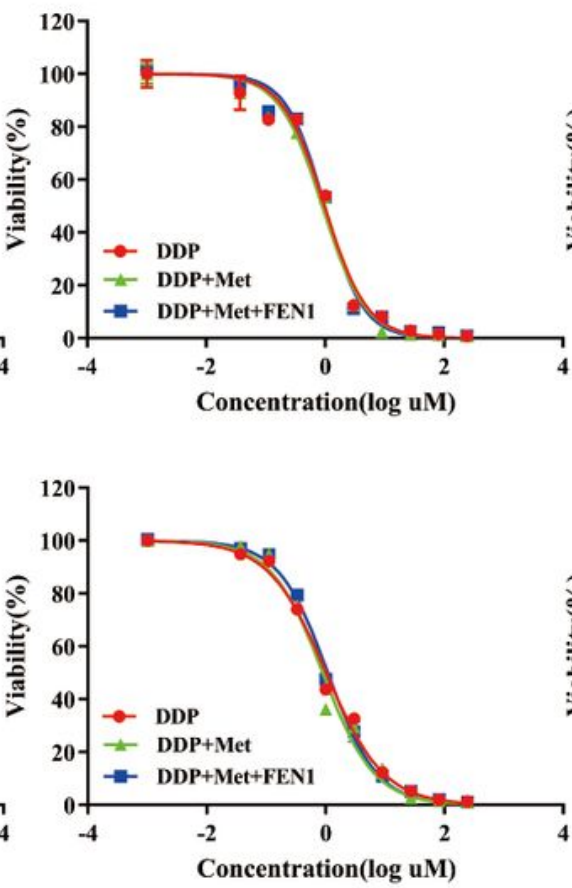

MTX
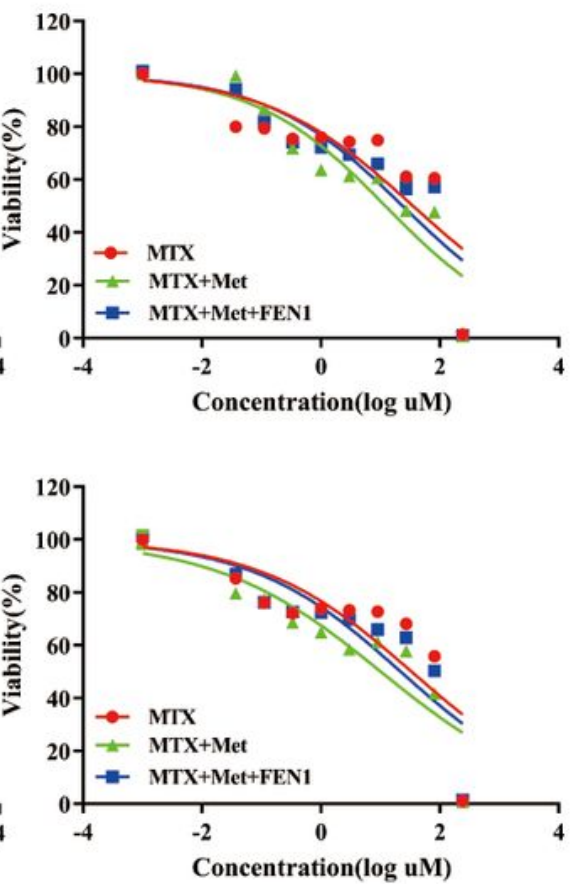

Figure 6

FEN1 participates metformin induced chemosensitivity modulation of OS cells. (A-C) Metformin promoted cytotoxic agents induced cell death in OS cells, which was compromised by FEN1 overexpression partly. ${ }^{\star} p<0.05,{ }^{\star} * p<0.01,{ }^{\star} * \star p<0.001$ vs. control. 
Figure 7

A
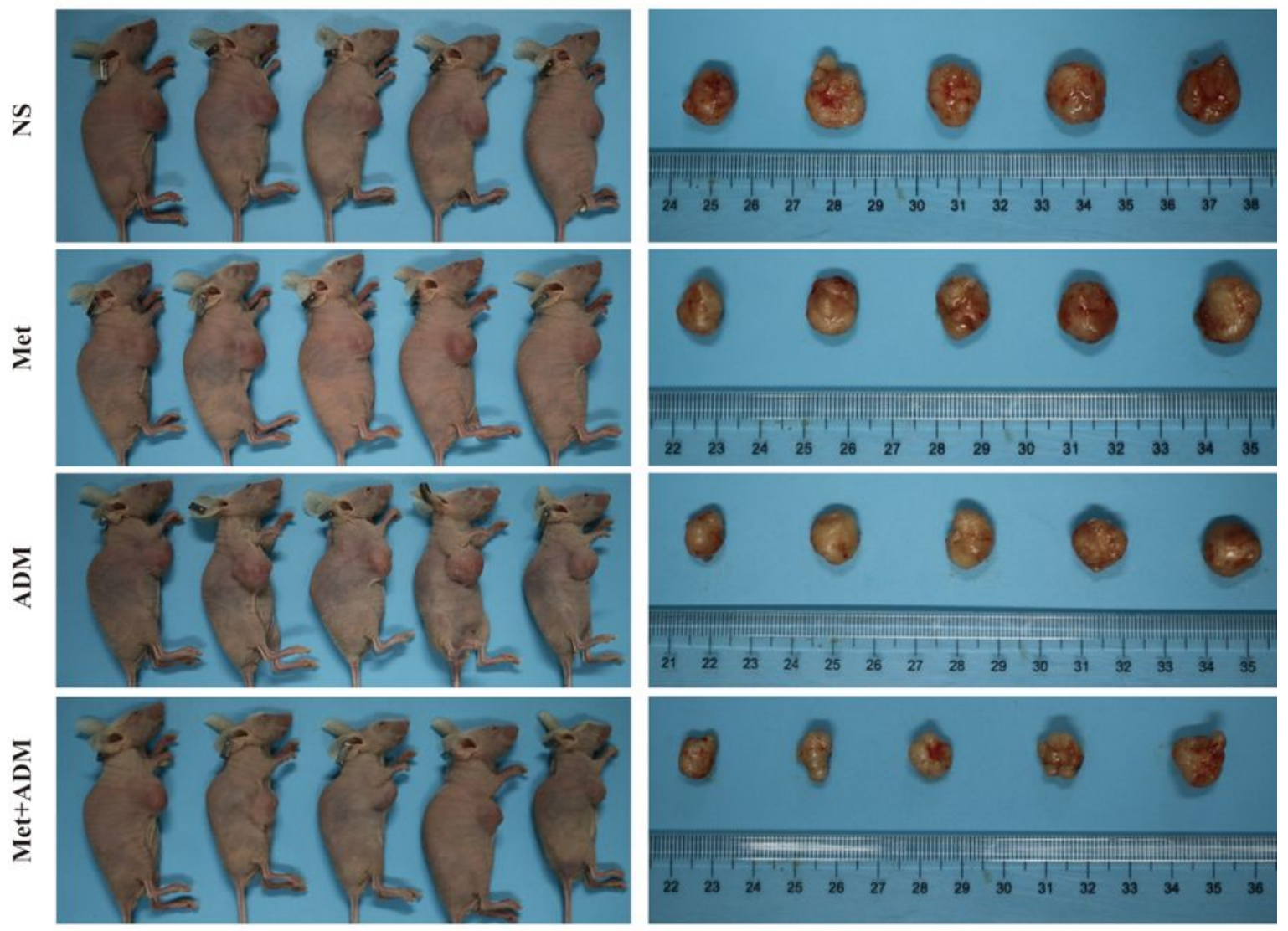

B
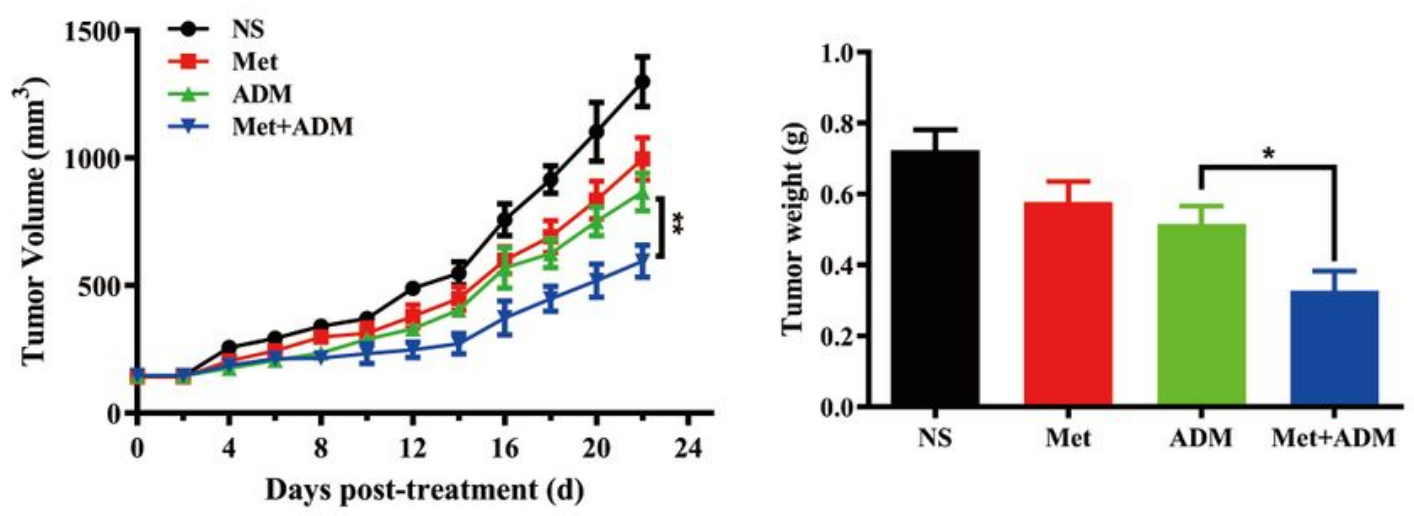

Figure 7

Metformin modulates chemosensitivity of OS cells in nude mice model. (A) Representative pictures show mice and tumors from each group at 21 days post-inoculation. $(B, C)$ Tumor volumes and weights in four groups over time. * $p<0.05, * \star p<0.01, * \star \star p<0.001$ vs. control. 